\title{
Perception of sleep in the elderly
}

\author{
Ståle Pallesen, Inger Hilde Nordhus and Geir Høstmark Nielsen \\ Departement of Clinical Psychology, University of Bergen, Norway \\ Correspondence to: Ståle Pallesen, Outpatient Clinic for Adults and the Elderly, Christiesgt. 12, 5015 Bergen \\ Telephone 55588842 Telefax $55589877 \quad$ E-mail staale.pallesen@psych.uib.no
}

\begin{abstract}
Background: Discrepancies between objectively and subjectively measured sleep variables make diagnosing insomnia in the elderly difficult. Also relevant to diagnosing insomnia in the elderly are expectations about sleep, gender and use of sedative-hypnotic medication. The present study focuses on how these variables relate to insomnia and sleep satisfaction.

Method: A questionnaire focusing on the subjective experience of sleep was administered to $116 \mathrm{older}$ (60 years and above) visitors at 4 senior centres in Bergen, Norway.

Results: For those generally satisfied with their sleep, mean sleep onset latency was 37 minutes and mean wake after sleep onset was 38 minutes. It was further demonstrated that $59.2 \%$ of the sample had unrealistic positive expectations (did not expect worsening of sleep with age) regarding sleep in old age. Those using sedative-hypnotic medication (23.3\%) were less satisfied with their sleep and felt less refreshed during the day than non-users. Contrary to most studies, no general gender differences in perception of sleep was revealed. The only exception was total sleep time where men reported more sleep than women ( 6.78 vs. 6.15 hours) per day.

Conclusion: The results cast doubts about the usefulness of the common criteria (30 minutes sleep onset latency and wake after sleep onset) used in clinical contexts to diagnose insomnia. Unrealistic positive expectations about sleep changes with age can lower the threshold for complaining and thus contribute to dissatisfaction and worry about sleep. Sedative-hypnotic drugs did seem to have limited benefit for the participants in this study.
\end{abstract}

Key Words: insomnia; older adults; diagnosis; perception of sleep

\section{INTRODUCTION}

An important point that is related to diagnosing insomnia is the individual expectations and beliefs about sleep. Unrealistic positive beliefs about the soundness of sleep can lower the threshold for complaining about insomnia. This concern is particularly warranted for the elderly (Morin et al., 1993) because sleep normally changes in a negative direction with advancing age. Increased sleep onset latency (SOL) and increased wake time after sleep onset (WASO) constitute normal age-related changes in sleep (Miles \& Dement, 1980). This may indicate that patients whose complaints are based on unrealistic sleep expectations, do not suffer from any sleep disorder. However, faulty appraisals may heighten emotional arousal and turn what are normal changes in sleep patterns into a clinical problem (Morin et al., 1993). It is also important to remember that subjective complaints and objective, that is polysomnographic (electroencephalogram, electroocculogram and electromyogram) recordings of sleep not always converge (e.g. Volk et al., 1991). Studies have also demonstrated large inter-individual differences in sleep parameters (McGhie \& Russel, 1962). Subjective reports on disturbed sleep, therefore, seem to be important as sleep quality in the first place is a subjective phenomenon.

Insomnia is the most common sleep disorder (Bixler et al., 1979) and its prevalence rises with age (Lacks, 1987). In clinical and research contexts alike, a common subdivision of insomnia is between sleep onset insomnia (difficulty falling asleep), sleep maintenance insomnia (difficulty getting back to sleep during night), and terminal insomnia (inability to return to sleep, even for a short while, after waking up during the night or early in the morning). For the two former types, sleep onset latency or wake time after sleep onset has to be greater than $30 \mathrm{~min} / \mathrm{night}$, with a minimum of 3 nights per week in order to be recognized as insomnia. For the latter type one usually requires total sleep time to be less than $6.5 \mathrm{hrs}$ per night (Lacks \& Morin, 1992). Patients who suffer from insomnia typically ask their general practitioner for drugs to facilitate sleep (Hohagen et al., 1994). Empirical studies demonstrate that short-term use of sedative-hypnotic drugs shortens sleep onset latency (SOL) and wake after sleep onset (WASO) and increases total sleep time (TST; Grad, 1995). If the same pattern could be 
observed on a long-term basis, one would expect older adults taking sleeping pills to become quite satisfied with their sleep. However, properly controlled studies of the long-term effects of sedative-hypnotic medication in the elderly are still missing (Grad, 1995). Evidence does indicate, however, that there might be an association between long-term use of sedativehypnotic drugs on a regular basis and a reduction in deep sleep (Schneider-Helmert, 1988). A reduction in deep sleep will lower the threshold for awakening and make the sleep more fragmented. If this holds true, one might expect elderly users of hypnotics to be unsatisfied with their sleep. Another variable related to complaints of sleep difficulties is gender. Studies have shown that women complain of insomnia more often than men (Brabbins et al., 1993), despite lack of corresponding differences in objective sleep measures like polysomnography (Hoch et al., 1987).

Thus, in evaluating sleep complaints in the elderly patient, several factors must be taken into consideration, some of which are addressed in the present paper, by the following questions:

1) What expectations do people have about sleep changes with increasing age?

2) Are the current diagnostic criteria of minimum 30 min sleep latency and 30 min time awake after sleep onset for sleep onset insomnia and sleep maintenance insomnia reasonable in understanding sleep complaints in old age?

3) What is the relationship between use of sedativehypnotic mediaction and satisfaction with sleep ?

4) Is there a relationship between gender and sleep expectations and perceptions?

In order do explore these questions, a sleep questionnaire was constructed reflecting central variables concerning sleep.

\section{METHODS}

Four senior centres in Bergen were contacted and asked for permission to invite elderly visitors to participate in a study of sleep. Permission was given in all instances and the sleep questionnaire was administered to visitors age 60 and above. All participants were allowed to ask for assistance in completing the questionnaire. In case of assistance this was given by the first author by explaining and elaborating the items. Based on information from the staff, the questionnaire was not given to elderly with any indications of cognitive impairment.

The form administered comprised 14 questions. Written information about the content of the questions emphasised that some of the questions might be difficult to answer in an exact way, and that there were no generally "right" or "wrong" answers. The content of the questions is given in Table 1. Four questions were related to demography, while 10 questions concerned various sleep variables. Central variables were: total sleep time (TST), sleep onset latency (SOL), and wake after sleep onset (WASO). The questionnaire also asked about perception of required amount of sleep, general satisfaction with actual sleep (7 point Likert scale), and eventual daytime sedation (7 point Likert scale). Subjects with a score of 1, 2 or 3 on the sleep satisfaction item were classified as unsatisfied with their sleep; subjects with a score of 4 were classified as neither satisfied nor unsatisfied; and subjects with a score of 5, 6 or 7 were classified as satisfied with their sleep. The questionnaire also contained an item about the use of sedative-hypnotic drugs (regularly or intermittent). Type of medication was not specified. In order to assess the informants' understanding of the general worsening of sleep with age, this issue was also included in the questionnaire. Cases with missing data were excluded from the data analysis.

Table 1. Topics addressed in the questionnaire.

\begin{tabular}{rll}
\hline Item & Topic & Response mode \\
\hline 1. & Age & no predefined class \\
2. & Sex & male/female \\
married/unmarried/divorced/widow(er)/cohabiter \\
3. & Marital status & living alone/not alone \\
4. & Living arrangement & no predefined class \\
5. & Sleep time needed in order to feel refreshed in the morning & no predefined class \\
6. & Sleep time usually obtained per day & no predefined class \\
7. & Sleep time obtained yesterday & no predefined class \\
8. & Common sleep onset latency & no predefined class \\
9. & Common wake time after sleep onset & $1=$ "very unsatisfied" $-7=$ "very satisfied" \\
10. & General satisfaction with sleep & $1=$ "very tired" $-7=$ "very refreshed" \\
11. & Feeling refreshed during the day & yes/no \\
12. & Present use of hypnotics & $1=$ "worsening", $2=$ "no change", $3=$ "improvement" \\
13. & Expectation of change in sleep with age & yes/no \\
14. & Special responses conducted in order to improve sleep & \\
\hline
\end{tabular}


Table 2. Frequency, means and standard deviations (in parentheses) of central sleep variables.

\begin{tabular}{lcccc}
\hline Sleep satisfaction & Percent & SOL (min.) & WASO (min.) & TST (hours) \\
\hline Not satisfied & 19.1 & $62.5(59.3)$ & $65.5(69.4)$ & $5.40(1.55)$ \\
In between & 11.5 & $54.1(60.8)$ & $99.8(70.5)$ & $5.69(1.42)$ \\
Satisfied & 69.6 & $37.0(32.1)$ & $38.0(57.1)$ & $6.86(1.22)$ \\
\hline
\end{tabular}

$\mathrm{SOL}=$ sleep onset latency, WASO $=$ wake after sleep onset, $\mathrm{TST}=$ total sleep time

\begin{tabular}{lccc}
\hline \multicolumn{2}{l}{ Use of hypnotics } & Sleep satisfaction $(1-7)$ & Feeling refreshed (1-7) \\
\hline Yes & 23.3 & $3.88(1.61)$ & $3.44(1.22)$ \\
No & 76.7 & $5.48(1.46)$ & $4.90(1.66)$ \\
\hline
\end{tabular}

\begin{tabular}{lc}
\hline Expectations about changes in sleep with increasing age \\
\hline Worsening & 40.9 \\
No change & 52.2 \\
Improvement & 7.0 \\
\hline
\end{tabular}

\section{RESULTS}

The total sample consisted of 116 older adults (mean age $77.1, \mathrm{SD}=6.30$, range 62-97 years), 64 females (mean age $77.4, \mathrm{SD}=6.53$ ) and 52 males (mean age 76.3, $\mathrm{SD}=6.04)$. Table 2 shows frequencies, means and standard deviations for central sleep variables studied.

\section{Expectations about sleep changes with age}

Of the total sample $40.9 \%$ expected worsening of sleep with advancing age, $52.2 \%$ expected no changes in sleep with age, while $7.0 \%$ expected sleep to improve with age. Subjects who used hypnotic medications expected to a greater extent than non-users the sleep to worsen with age $(\mathrm{t}=-5.17, \mathrm{df}=114, \mathrm{p}<.001)$.

\section{Relationship between perception of different sleep parameters and general satisfaction with sleep}

Of the total sample $19.1 \%$ were unsatisfied with their sleep, $11.3 \%$ were neither unsatisfied nor satisfied with their sleep and $69.6 \%$ were satisfied. Of the subjects who where satisfied with their sleep, $37.0 \%$ reported sleep onset latency scores of 30 minutes or more, compared to of $55.0 \%$ of the subjects that were unsatisfied with their sleep. The mean SOL values for the groups are reported in Table 2. Furthermore, $25.6 \%$ of the subjects who were satisfied with their sleep had WASO scores above 30 minutes, while the corresponding figure for unsatisfied sleepers was $52.4 \%$. The mean WASO scores for the two groups are also reported in Table 2 .

\section{Relationship between use of sedative-hypnotic medication and satisfaction with sleep}

Sedative-hypnotic drugs were used by $23.3 \%$ of the sample, while $76.7 \%$ did not take any hypnotic medication at the time of observation. T-tests were conducted in order to investigate the relationship between use of medications and satisfaction with sleep. Hypnotic users were less satisfied with their sleep than non-users $(\mathrm{t}=-4.79, \mathrm{df}=113, \mathrm{p}<.001)$. Users also felt less refreshed during the day compared to nonusers $(\mathrm{t}=-4.22, \mathrm{df}=114, \mathrm{p}<.001)$.

\section{Relationship between gender and sleep expectations and perceptions}

As reported in Table 3, there were no significant gender differences related to: satisfaction with sleep, feeling of being refreshed during the day, expectations of changes in sleep quality with age, SOL, WASO and required amount of sleep. However, a significant gender difference was found in TST-scores, where men reported larger total sleep time compared to women.

\section{DISCUSSION}

About $60 \%$ of the sample did not expect worsening of sleep contingent upon increasing age. In turn this may lower an individuals threshold for being unsatisfied with the sleep. The reported finding might also be related to the American Sleep Disorder Association category: subjective (or sleep misperception) insomnia, or so-called pseudoinsomnia (American Sleep Disor- 
Table 3. Gender differences in self-reported sleep variables (means and standard deviations).

\begin{tabular}{lccrc}
\hline Variable & Women & Men & $\mathrm{t}$ & $\mathrm{p}$ \\
\hline Sleep satisfaction (1-7) & $5.03(1.67)$ & $5.23(1.59)$ & -0.65 & 0.52 \\
Refreshed during the day (1-7) & $4.41(1.69)$ & $4.75(1.67)$ & -1.10 & 0.28 \\
Expectations of changes (1-3) & $1.67(0.65)$ & $1.65(0.56)$ & 0.11 & 0.92 \\
Sleep onset latency (min) & $47.4(43.6)$ & $39.9(42.2)$ & 0.92 & 0.36 \\
Wake after sleep onset (min) & $50.6(59.6)$ & $49.7(68.6)$ & 0.08 & 0.94 \\
Sleep amount needed (hours) & $6.91(1.18)$ & $7.23(0.93)$ & -1.54 & 0.13 \\
Total sleep time (hours) & $6.15(1.54)$ & $6.78(1.25)$ & -2.37 & 0.02 \\
\hline
\end{tabular}

ders Association, 1990). The main criteria for this disorder is subjective sleep complaints not corroborated by polysomnographic data. In line with this way of thinking some individuals can be said to overestimate their sleep difficulties. The reverse situations will be represented by those who underestimate their sleep problems. Lichstein and Johnson (1991) report that even non-complaining older sleepers showed mean WASO scores, measured with polysomnography (PGS), higher than 30 minutes. Our data indicate that the common 30 minutes criteria of SOL and WASO used in diagnosing sleep onset and sleep maintenance insomnia respectively, to a very small degree differentiate between individuals who are satisfied vs. unsatisfied with their sleep. The discrepancies between estimates of sleep parameters of satisfied and unsatisfied sleepers are more or less marginal. This finding may be associated with individual variability in sleep requirements (McGhie \& Russel, 1962). A person who feels refreshed after 5 hours of sleep will not complain if he or she obtains this amount of sleep, but the reverse will probably be true for a person who needs 7 hours of sleep to feel refreshed. Individual variability has to a small extent been taken into consideration when diagnosing insomnia (Trinder, 1988).

The frequent use sedative-hypnotic medication among the elderly is confirmed in this study, where approximately one in four reported using such drugs, either on an intermittent or continuous basis. Sedativehypnotic drug users demonstrated more realistic expectations about sleep compared to non-users. This may indicate that experiencing bad sleep can shape expectations about sleep in an more realistic manner. If sedative-hypnotic drugs work well one would expect hypnotic users to be quite satisfied with their sleep. The results indicate, however, that sedative-hypnotic drug users were less satisfied with their present sleep compared to non-users. This finding can probably not be attributed to unrealistic sleep expectations. An alternative hypothesis is that medication do not have the desired effect, since deep sleep stages deteriorates contingent upon long-term use of these drugs (Schneider-Helmert, 1988).
The results also demonstrated that hypnotic users felt less refreshed during the day, compared to nonusers. This may be related to daytime sedation, a welldocumented side effect of hypnotic use (Institute of Medicine, 1979). A tentative conclusion is that sedative-hypnotic drugs can maintain and in fact further enhance the symptoms of insomnia, which in turn indicates that the distinction between primary insomnia and insomnia associated with drug or alcohol dependency can be blurred.

The final question addressed in the present study concerned the relationship between gender and sleep difficulties. Contrary to former studies (Brabbins et al., 1993) gender difference did not emerge, except for total sleep time where men reported longer total sleep time than women. Why the expected gender difference did not reveal itself in this study is unclear. A hypothesis might be that gender differences do not occur in all sub-samples of elderly people. Further studies therefore should address this question more directly.

An obvious limitation of the present study is the retrospective approach to data collection. Such data yields primarily global and impressionistic descriptions about the sleep of the older adults. The validity of the data can be susceptible to potential mood and memory biases. Another problem is that the data are only estimates of average values for each individual. Hence, they do not comprise the intra-individual variability of the included sleep parameters, that most certainly may have added valuable information to the questions addressed in the present study (Espie, 1991). Although the elderly included in the study cannot be regarded as representative for elderly in general, the data still illuminates some central topics related to the perception of sleep in the elderly.

\section{CONCLUSION}

The primary focus of this study was to address subjective perception of sleep in older adults. It is concluded that subjective sleep rating should be more strongly emphasised in diagnosing and evaluating 
complaints of insomnia. The main reason for this is that individual perceptions of sleep not only represent a symptom, but probably also act as causative factors in the development of insomnia. Many challenges confront those measuring sleep, but one of the more important ones is the source of information used to assess sleep. Attention should also be paid to sedativehypnotic drugs as another potential causative factor in development of insomnia. It should be remembered that disturbed sleep as reported by the individual is the basis upon which prescription for sedative-hypnotic medication is written.

\section{REFERENCES}

American Sleep Disorders Association. The international classification of sleep disorders: Diagnostic and coding manual. Rochester, MN: Arthor, 1990.

Bixler EO, Kales A, Soldatos CR, et al. Prevalence of sleep disorders in the Los Angeles metropolitan area. Am J Psychiatry 1979; 136: 1257-1262.

Brabbins CJ, Dewey ME, Copeland JRM, et al. Insomnia in the elderly: Prevalence, gender differences and relationships with morbidity and mortality. Int J Geriatr Psychiatry 1993; 8: 473-480.

Espie CA. The psychological treatment of insomnia. Chichester: John Wiley \& Sons, 1991.

Grad RM. Benzodiazepines for insomnia in community-dwelling elderly: A review of benefit and risk. $J$ Fam Pract 1995; 41: 473-481.

Hoch CC, Reynolds CF III, Kupfer DJ, et al. Empirical note: Self-report versus recorded sleep in healthy seniors. Pychophysiology 1987; 24: 293-299.

Hohagen F, Käppler C, Schramm E, et al. Prevalence of insomnia in the elderly general practice attenders and the current treatment modalities. Acta Psychiatr Scand 1994; 90: 102-108.

Institute of Medicine. Sleeping pills, insomnia, and medical practice. Washington, DC: National Academy of Sciences, 1979.

Lacks P. Behavioral treatment for persistent insomnia. New York: Pergamon Press, 1987.

Lacks P, Morin CM. Recent advances in the assessment and treatment of insomnia. J Consult Clin Psychol 1992; 60: $586-594$.

Lichstein KL, Johnson RS. Older adults' objective self-recording of sleep in the home. Behav Ther 1991; 22: 531548.

McGhie A, Russel SM. The subjective assessment of normal sleep patterns. J Ment Sci 1962; 108: $642-654$.

Miles LE, Dement WC. Sleep and aging. Sleep 1980; 3: 119-220.

Morin CM, Stone J, Trinkle D, et al. Dysfunctional beliefs and attitudes about sleep among older adults with and without insomnia complaints. Psychol Aging 1993; 8: 463-467.

Schneider-Helmert D. Why low-dose benzodiazepine-dependent insomniacs can't escape their sleeping pills. Acta Psychiatr Scand 1988; 78: 706-711.

Trinder J. Subjective insomnia without objective findings: A pseudo diagnostic classification? Psychol Bull 1988; 103: 87-94.

Volk S, Jost K, Blass K, et al. Untersuchungen zum schlaf-wachverhalten hochbetrager. Z Gerontol 1991; 24: 203-210. 